\title{
Optimizing future experiments of cosmic far-infrared background: a principal component approach
}

\author{
Hao-Yi Wu ${ }^{1,2 \star}$ and Olivier Doré ${ }^{1,2 \star}$ \\ ${ }^{1}$ California Institute of Technology, MC 367-17, Pasadena, CA 91125, USA \\ ${ }^{2}$ Jet Propulsion Laboratory, California Institute of Technology, 4800 Oak Grove Drive, Pasadena, CA 91109, USA
}

Accepted 2017 February 9. Received 2016 December 8; in original form 2016 December 8

\begin{abstract}
The anisotropies of cosmic far-infrared background (CFIRB) probe the star formation rate (SFR) of dusty star-forming galaxies as a function of dark matter halo mass and redshift. We explore how future CFIRB experiments can optimally improve the SFR constraints beyond the current measurements of Planck. We introduce a model-independent, piecewise parametrization for SFR as a function of halo mass and redshift, and we calculate the Fisher matrix and principal components of these parameters to estimate the SFR constraints of future experiments. We investigate how the SFR constraints depend on angular resolution, number and range of frequency bands, survey coverage and instrumental sensitivity. We find that the angular resolution and the instrumental sensitivity play the key roles. Improving the angular resolution from 20 to 4 arcmin can improve the SFR constraints by 1.5-2.5 orders of magnitude. With the angular resolution of Planck, improving the sensitivity by 10 or 100 times can improve the SFR constraints by one or two orders of magnitude, and doubling the number of frequency bands can also improve the SFR constraints by an order of magnitude. We find that survey designs like the Cosmic Origins Explorer (CORE) are very close to the optimal design for improving the SFR constraints at all redshifts, while survey designs like LiteBIRD and CMB-S4 can significantly improve the SFR constraints at $z \gtrsim 3$.
\end{abstract}

Key words: methods: statistical-galaxies: haloes-galaxies: star formation-cosmology: theory-submillimetre: diffuse background-submillimetre: galaxies.

\section{INTRODUCTION}

Cosmic far-infrared background $(\mathrm{CFIRB})^{1}$ originates from unresolved, dusty star-forming galaxies across cosmic time. In dusty star-forming galaxies, newly formed massive stars produce abundant ultraviolet (UV) photons, and $\sim 90$ per cent of these UV photons are absorbed by the interstellar dust, which is heated to approximately $15-60 \mathrm{~K}$ and emits nearly blackbody radiation in infrared (IR), with a peak at $\sim 60-100 \mu \mathrm{m}(3000-5000 \mathrm{GHz})$ in the rest frame. At high redshift, these galaxies are observed in far-infrared (FIR) and submillimetre (submm) bands. These FIR/submm observations of galaxies probe their star formation rate (SFR) and are highly complementary to UV observations (e.g. Casey, Narayanan \& Cooray 2014; Lutz 2014; Madau \& Dickinson 2014). However, the majority of the FIR/submm galaxies are unresolved due to the low resolution of telescopes at these wavelengths. Therefore, CFIRB provides a unique way to reveal the star-forming activ-

\footnotetext{
^ E-mail: hywu@ @altech.edu (H-YW); Olivier.P.Dore@jpl.nasa.gov (OD)

${ }^{1}$ We use the longer acronym CFIRB instead of CIB, because the latter sometimes refers to cosmic near-infrared background, which originates from old stellar populations.
}

ities under the current resolution limit. In particular, the anisotropies of CFIRB probe how SFR is related to the underlying dark matter haloes.

The observability of CFIRB was first predicted by Bond, Carr \& Hogan (1986). Since its discovery by COBE (Puget et al. 1996; Fixsen et al. 1998; Hauser et al. 1998; Hauser \& Dwek 2001), and subsequent observations of ISO (Elbaz et al. 2002) and Spitzer (Dole et al. 2006; Lagache et al. 2007), CFIRB has opened a new window for observing the star formation activities in high-redshift, low-mass galaxies (e.g. Chary \& Elbaz 2001; Lagache, Puget \& Dole 2005). More recently, the measurements of CFIRB anisotropies have been significantly improved by BLAST (Viero et al. 2009), SPT (Hall et al. 2010), AKARI (Matsuura et al. 2011), ACT (Hajian et al. 2012), Herschel (Amblard et al. 2011; Berta et al. 2011; Viero et al. 2013) and Planck (Planck Collaboration XVIII 2011; Planck Collaboration XXX 2014). These measurements have enabled detailed modelling of the galaxy populations contributing to CFIRB (e.g. De Bernardis \& Cooray 2012; Shang et al. 2012; Xia et al. 2012; Addison, Dunkley \& Bond 2013; Béthermin et al. 2013; Thacker et al. 2013; Planck Collaboration XXX 2014; Cowley et al. 2016; Wu \& Doré 2017; Wu, Doré \& Teyssier 2016).

The next-generation space-borne missions of cosmic microwave background (CMB) will include FIR/submm bands to measure dust 
emissions and will significantly improve CFIRB measurements. These missions include the Cosmic Origins Explorer (CORE; The COrE Collaboration 2011; De Zotti et al. 2015, 2016; Di Valentino et al. 2016), the Primordial Inflation Explorer (PIXIE; Kogut et al. 2011), the LiteBIRD (Matsumura et al. 2014) and the Experimental Probe of Inflationary Cosmology (EPIC, Bock et al. 2009). In addition, the next-generation the Stage-4 ground-based cosmic microwave background experiment (CMB-S4, Abazajian et al. 2016) will have superior resolution and sensitivity, which are essential for improving CFIRB measurements. The CFIRB measurements from these missions will have the potential to constrain the cosmic star formation history to high accuracy. With the planning of these missions underway, it is imperative to understand the optimal survey designs for constraining the cosmic star formation history.

In this work, we explore how future CFIRB experiments can most effectively constrain the cosmic star formation history. We introduce a model-independent, piecewise parametrization for SFR of galaxies as a function of halo mass and redshift, and we calculate the Fisher matrix and principal components of these parameters to assess the SFR constraints. We explore the impact of angular resolution, number and range of frequency bands, survey coverage and instrumental sensitivity. We find that the angular resolution and the instrumental sensitivity are the two main factors for determining the SFR constraints from an experiment. For example, an angular resolution improvement from 20 to 4 arcmin can lead to 1.5-2.5 orders of magnitude improvement in SFR constraints. With the angular resolution of Planck, increasing the sensitivity by 10 times (as in the case of CORE) or 100 times (as in the cases of LiteBIRD and CMB-S4) with respect to Planck will lead to one to two orders of magnitude improvement in SFR constraints.

In addition, we find that increasing the number of frequency bands is not always effective in improving SFR constraints. For example, with the angular resolution of Planck, doubling the number of frequency bands of Planck can improve the SFR constraints by approximately an order of magnitude, but further increasing the number of bands has less significant impact. However, if we degrade the angular resolution to $20 \mathrm{arcmin}$, increasing the number of bands leads to limited improvement in SFR constraints. Comparing our results with the designs of several future surveys, we find that the survey designs of CORE are very close to optimal for constraining SFR at all redshifts, while the survey designs of LiteBIRD and CMB-S4 can provide valuable SFR constraints at $z \gtrsim 3$.

This paper is organized as follows. In Section 2, we review the halo model for calculating the CFIRB angular power spectra. In Section 3, we introduce our model-independent, piecewise parametrization for SFR, as well as the Fisher matrix and the principal component approach. Section 4 explores how SFR constraints depend on angular resolution, number of frequency bands, sky coverage and instrumental sensitivity. Section 5 studies the optimal range of frequency bands. We discuss the implications of our results for future missions in Section 6, and we summarize in Section 7.

Following Planck Collaboration XXX (2014), we adopt a flat $\Lambda$ CDM cosmology with the following cosmological parameters: $\Omega_{\mathrm{m}}=0.3175 \Omega_{\Lambda}=0.6825 ; \Omega_{\mathrm{b}} h^{2}=0.022068 ; \sigma_{8}=0.8344$. $h=0.6711 ; n_{\mathrm{s}}=0.9624$. The halo mass in this work refers to the virial mass.

\section{MODELLING THE CFIRB POWER SPECTRA}

We adopt the halo model implementation in Shang et al. (2012, S12 thereafter) and in the Planck 2013 results (Planck Collaboration XXX 2014, P13 thereafter) as our fiducial model to calculate the

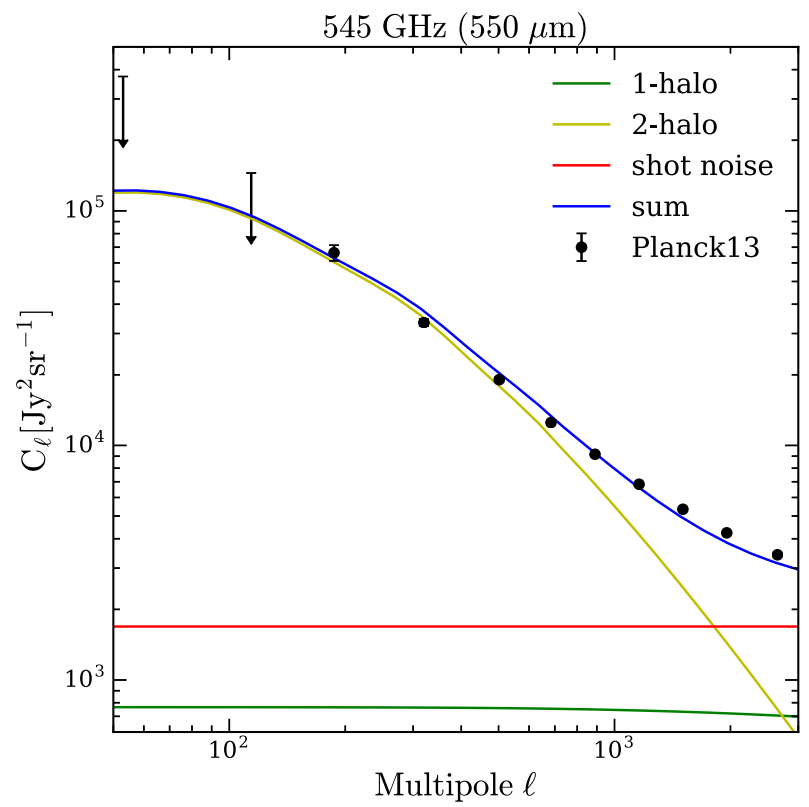

Figure 1. Angular power spectrum of CFIRB at $545 \mathrm{GHz}(550 \mu \mathrm{m})$. The theory prediction (blue) is broken down into the 1-halo term (green), the 2-halo term (yellow), and the shot noise (red). The black points are the observational results from P13 (given by their table D.2).

angular power spectra of CFIRB. This particular choice does not impact our results because we assess the SFR constraints in a modelindependent way (see Section 3 ). ${ }^{2}$ Our calculation includes the following steps:

(i) Modelling the SFR and the IR spectral luminosity as a function of halo mass and redshift (Section 2.1),

(ii) Calculating the 1-halo and 2-halo contributions to the CFIRB power spectra (Section 2.2),

(iii) Calculating the shot noise (Section 2.3),

(iv) Estimating the detector noise (Section 2.4).

Fig. 1 demonstrates the halo model prediction of the CFIRB angular power spectrum at $545 \mathrm{GHz}(550 \mu \mathrm{m})$, which agrees well with the observational results in P13. Below we describe our implementation in detail.

\subsection{IR luminosities and spectral energy distributions of galaxies}

One of the main goals of CFIRB experiments is to constrain SFR as a function of halo mass and redshift. We adopt the common assumption that the SFR is proportional to the IR luminosity,

$\operatorname{SFR}(M, z)=K L_{\mathrm{IR}}(M, z)$,

where $K=1.7 \times 10^{10} \mathrm{M}_{\odot} \mathrm{yr}^{-1} \mathrm{~L}_{\odot}$ based on the Salpeter initial mass function (Kennicutt 1998). Following P13, we assume that $L_{\mathrm{IR}}(M, z)$ is parametrized as

$L_{\mathrm{IR}}(M, z)=L_{0} \Phi(z) \Sigma(M)$,

\footnotetext{
${ }^{2}$ In Wu et al. (2016) and Wu \& Doré (2017), we develop physical and empirical models for interpreting CFIRB and other FIR/submm observables. These models help us interpret CFIRB physically and explore the consistency between FIR, optical and UV observations.
} 
where the redshift dependence is given by

$\Phi(z)=(1+z)^{\delta}$,

and the mass dependence is given by

$\Sigma(M)=\frac{M}{\left(2 \pi \sigma_{M}^{2}\right)^{1 / 2}} \exp \left\{-\frac{\left(\log _{10} M-\log _{10} M_{\mathrm{eff}}\right)^{2}}{2 \sigma_{M}^{2}}\right\}$.

We adopt the best-fitting values in table 9 in P13: $\delta=3.6$; $M_{\text {eff }}=10^{12.6} \mathrm{M}_{\odot} ; \sigma_{M}^{2}=0.5 ; L_{0}=0.0135 \mathrm{~L}_{\odot}$.

To predict the observed CFIRB, we need the spectral luminosity of galaxies, $L_{v}(M, z)$. We assume that the spectral energy density (SED), $\Theta_{v}$, depends only on redshift and is independent of $M$ and $L_{\mathrm{IR}}$; thus,

$L_{v}(M, z)=L_{\mathrm{IR}}(M, z) \Theta_{v}(z)$.

We assume that the SED is parametrized separately for low frequency (modifying the Rayleigh-Jeans law) and high frequency (modifying the Wien's law to a shallower plower law),

$\Theta_{v} \propto\left\{\begin{array}{ll}v^{\beta} B_{v}\left(T_{\mathrm{d}}\right) & \text { for } v<v_{0} \\ A v^{-\gamma} & \text { for } v \geq v_{0}\end{array}\right.$,

where $B_{v}$ is the Planck function. The factor $A$ and the peak frequency $v_{0}$ are calculated by imposing the continuity of $\Theta(v)$ and $d \Theta / d \nu$ at $v=v_{0}$. The SED is normalized such that $\int \Theta_{v} \mathrm{~d} v=1$; we integrate between 100 and $10^{6} \mathrm{GHz}$, which has been tested to ensure convergence. We assume that the dust temperature $T_{\mathrm{d}}$ depends only on redshift,

$T_{\mathrm{d}}=T_{0}(1+z)^{\alpha}$.

We again adopt the best-fitting values in table 9 in P13: $T_{0}=24.4 \mathrm{~K}$; $\alpha=0.36 ; \beta=1.75 ; \gamma=1.7$.

\subsection{CFIRB angular power spectra}

The mean emission coefficient of CFIRB is given by

$\bar{j}_{v}(z)=\int \mathrm{d} M \frac{\mathrm{d} n}{\mathrm{~d} M}\left[f_{v}^{\mathrm{cen}}(M, z)+f_{v}^{\mathrm{sat}}(M, z)\right]$,

where $f_{v}^{\text {cen }}$ and $f_{v}^{\text {sat }}$ correspond to the contribution from central and satellite galaxies, respectively,

$$
\begin{aligned}
f_{v}^{\text {cen }}(M, z) & =\frac{1}{4 \pi} N_{c} L_{(1+z) v}(M, z), \\
f_{v}^{\text {sat }}(M, z) & =\frac{1}{4 \pi} \int_{M_{\min }}^{M} \mathrm{~d} M_{s} \frac{\mathrm{d} N}{\mathrm{~d} M_{s}}(M) L_{(1+z) v}\left(M_{s}, z\right) .
\end{aligned}
$$

In the equations above, $(1+z) v$ is the rest-frame frequency; we assume that central and satellite galaxies follow the same $L_{v}(M, z)$; $\mathrm{d} n / \mathrm{d} M$ is the halo mass function, and we adopt the fitting function in Tinker et al. (2010); $N_{c}(M)$ determines whether a halo of mass $M$ hosts a central galaxy, and $N_{c}=1$ if $M \geq M_{\min }$ and 0 otherwise; we assume $M_{\min }=10^{10} \mathrm{M}_{\odot} ; \mathrm{d} N / \mathrm{d} M_{s}(M, z)$ is the number of subhaloes of mass $M_{s}$ in a central halo of mass $M$, and we adopt the fitting function in Tinker \& Wetzel (2010).

The $3 \mathrm{D}$ auto and cross power spectrum of $\left(j_{v}, j_{v^{\prime}}\right)$ is given by the sum of the 2-halo and the 1-halo terms,

$P_{v v^{\prime}}(k, z)=P_{v v^{\prime}}^{2 \mathrm{~h}}(k, z)+P_{v v^{\prime}}^{1 \mathrm{~h}}(k, z)$.

The 2-halo term is contributed by galaxy pairs from two distinct haloes and is proportional to the linear matter power spectrum $P_{\text {lin }}(k)$,

$P_{\nu v^{\prime}}^{2 \mathrm{~h}}(k, z)=\bar{b}_{\nu}(z) \bar{b}_{v^{\prime}}(z) P_{\mathrm{lin}}(k, z)$, where $\bar{b}_{v^{\prime}}(z)$ is the effective galaxy bias calculated by integrating the halo bias $b(M, z)$,

$\bar{b}_{v}=\frac{1}{\bar{j}_{v}} \int \mathrm{d} M \frac{\mathrm{d} n}{\mathrm{~d} M} b(M, z)\left(f_{v}^{\mathrm{cen}}+f_{v}^{\mathrm{sat}}\right)$.

We use the linear matter power spectrum calculated by CAMB (Lewis, Challinor \& Lasenby 2000) and the fitting function of halo bias in Tinker et al. (2010).

The 1-halo term is contributed by galaxy pairs in the same halo,

$$
\begin{aligned}
P_{\nu v^{\prime}}^{1 \mathrm{~h}}(k, z)= & \frac{1}{\bar{j}_{v} \bar{j}_{v^{\prime}}} \int \mathrm{d} M \frac{\mathrm{d} n}{\mathrm{~d} M} \\
& \times\left(f_{v}^{\mathrm{cen}} f_{v^{\prime}}^{\mathrm{sat}} u+f_{v^{\prime}}^{\mathrm{cen}} f_{v}^{\mathrm{sat}} u+f_{v}^{\mathrm{sat}} f_{v^{\prime}}^{\mathrm{sat}} u^{2}\right),
\end{aligned}
$$

where $u(M, k)$ is the halo mass density profile in Fourier space; we use the NFW profile (Navarro, Frenk \& White 1997) with the concentration-mass relation from Bhattacharya et al. (2013).

With Limber approximation, the angular power spectrum of the CFIRB emission is given by

$C_{\ell, v v^{\prime}}^{\text {halo }}=\int \frac{\mathrm{d} z}{\chi^{2}} \frac{\mathrm{d} \chi}{\mathrm{d} z} a^{2} \bar{j}_{v}(z) \bar{j}_{\nu^{\prime}}(z) P_{\nu v^{\prime}}\left(k=\frac{\ell}{\chi}, z\right)$,

where $\chi(z)$ is the comoving distance.

Fig. 1 shows the 1-halo and the 2-halo contributions to the angular power spectrum at $545 \mathrm{GHz}(550 \mu \mathrm{m})$. As can be seen, the 2-halo term dominates most of the angular scales, and the 1-halo term is lower than the shot noise at all scales. For the CFIRB observed by Planck, the 1-halo term is subdominant for all frequency bands and angular scales (see fig. 12 in P13).

\subsection{Shot noise}

The shot noise of the power spectrum corresponds to self-pairs of galaxies. Since a galaxy can appear in different bands, the shot noise exists in both auto and cross power spectra. The shot noise is calculated by integrating the spectral flux function (also known as the number counts), $\mathrm{d} n / \mathrm{d} S_{v}$. The spectral flux is related to the spectral luminosity via

$S_{v}=\frac{L_{(1+z) v}}{4 \pi \chi^{2}(1+z)}$.

The shot noise is given by

$C_{v v^{\prime}}^{\text {shot }}=\int \mathrm{d} V \int \mathrm{d} S_{v} \frac{\mathrm{d} n}{\mathrm{~d} S_{v}} S_{v} S_{v^{\prime}}$

We present the detailed derivation in Appendix A. The red horizontal line in Fig. 1 demonstrates the shot noise level at $545 \mathrm{GHz}$ $(550 \mu \mathrm{m})$.

\subsection{Detector noise}

We assume that the detector noise only contributes to the auto power spectra. Based on Knox (1995), the angular power spectrum contributed by the detector noise is given by

$C_{\ell}^{\text {noise }}=w^{-1} \mathrm{e}^{\ell^{2} \sigma_{\mathrm{b}}^{2}}$,

where $w$ is the weight per solid angle,

$w^{-1}=\sigma_{\text {pix }}^{2} \Omega_{\text {pix }}$,

$\Omega_{\text {pix }}$ is the solid angle of the pixel,

$\Omega_{\mathrm{pix}}=\theta_{\mathrm{FWHM}}^{2}$, 
Table 1. Instrumental sensitivities adopted in this work. The values for Planck are taken from table 1 of P13 and table 6 of Planck Collaboration I (2014). In the last two columns, we assume hypothetical experiments with the same bandpass filters as Planck but different, frequency-independent $\sigma_{\text {pix }}\left(\right.$ in MJy sr $^{-1}$ ) and $\theta_{\text {FWHM }}$ (in arcmin). We assume various values for $\sigma_{\text {pix }}$ and $\theta_{\text {FWHM }}$ in Section 4 and Fig. 3, and the corresponding sensitivities can be estimated by rescaling the numbers in the last two columns.

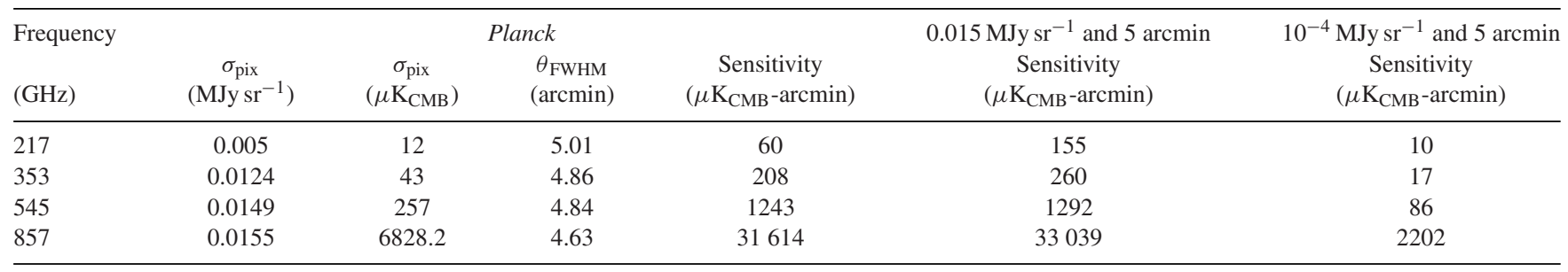

and $\sigma_{\mathrm{b}}$ is the beam size,

$\sigma_{\mathrm{b}}=\frac{\theta_{\mathrm{FWHM}}}{\sqrt{8 \ln 2}}$.

Here, $\sigma_{\text {pix }}$ is the detector noise per pixel and $\theta_{\text {FWHM }}$ is the full width at half-maximum of the Gaussian beam of a given band. In Table 1, we list the values of $\sigma_{\text {pix }}$ and $\theta_{\text {FWHM }}$ of Planck, as well as the sensitivity $w^{-1 / 2}=\sigma_{\text {pix }} \theta_{\text {FWHM }}$ in the unit of $\mu \mathrm{K}_{\mathrm{CMB}}$-arcmin.

In Sections 4 and 5, when calculating the constraints for future surveys, we assume that all frequency bands have the same $\theta_{\text {FWHM }}$ and $\sigma_{\text {pix }}$ (in the unit of $\left.\mathrm{MJy} \mathrm{sr}^{-1}\right)^{3}$ and that the highest multipole is determined by

$\ell_{\max }=\frac{\pi}{\theta_{\mathrm{FWHM}}}$.

In the last two columns of Table 1, we list the sensitivities of two hypothetical experiments with the same bandpass filters as Planck but different $\sigma_{\text {pix }}$ and $\theta_{\text {FWHM }}$. In Section 4 and Fig. 3, we will assume several combinations of $\sigma_{\text {pix }}$ and $\theta_{\text {FWHM }}$, and the corresponding sensitivities can be estimated by rescaling the numbers in the last two columns of Table 1 .

\section{FISHER MATRIX AND PRINCIPAL COMPONENT APPROACH}

In order to assess the SFR constraints of future experiments in a model-independent way, we adopt a piecewise parametrization for $\operatorname{SFR}(M, z)$ and calculate the Fisher matrix. The basic idea is as follows: for a given range of $M$ and $z$, we use a free parameter to describe the value of SFR in this range. We then calculate the Fisher matrix and the principal components of these parameters. The Fisher matrix indicates the information content of the parameters, and the first few principal components indicate the combinations of parameters that are best constrained. We note that this approach has been widely applied in cosmology (e.g. Huterer \& Starkman 2003; Mortonson \& Hu 2008).

\footnotetext{
${ }^{3}$ In the case of Planck, the $217 \mathrm{GHz}$ band has the lowest detector noise among the CFIRB bands (see Table 1). When we need to assume a frequencyindependent sensitivity, we use the detector noise of $545 \mathrm{GHz}(0.015$ $\mathrm{MJy} \mathrm{sr}^{-1}$ ) as a conservative baseline. We also note that the noise levels of 217 and $353 \mathrm{GHz}$ are calibrated in the unit of $\mu \mathrm{K}_{\mathrm{CMB}}$, while those of 545 and $857 \mathrm{GHz}$ are calibrated in the unit of $\mathrm{MJy} \mathrm{sr}^{-1}$. We use the conversion provided by $\mathrm{P} 13$, but we note that the conversion depends on the bandpass filter, which will be different for different experiments.
}

\subsection{Piecewise parametrization of SFR}

We assume a binned, piecewise parametrization for SFR,

$$
\begin{aligned}
\operatorname{SFR}(M, z)= & \sum_{i} \sum_{j}\left(1+f_{i j}\right) \operatorname{SFR}_{\mathrm{fid}}(M, z) \\
& \times H\left(M_{i}, M_{i+1}\right) H\left(z_{j}, z_{j+1}\right),
\end{aligned}
$$

where $H\left(x_{i}, x_{i+1}\right)$ is the top-hat function and equals 1 if $x_{i} \leq x$ $<x_{i+1}$ and 0 otherwise. The fiducial model $\operatorname{SFR}_{\text {fid }}(M, z)$ is described in Section 2.1. Each of the $f_{i j}$ parameter corresponds to the perturbation around the fiducial model in a mass and redshift bin. We adopt six redshift bins between $z=0$ and 6, and five logarithmic mass bins between $\log _{10} M=10$ and 15 ; this results in $30 f_{i j}$ parameters.

\subsection{Fisher matrix}

The Fisher matrix is the inverse of the covariance matrix of model parameters and is used to estimate the parameter constraints for a given data set. The Fisher matrix for the CFIRB angular power spectra is given by (see e.g. Knox et al. 2001):

$F_{\alpha \beta}=\sum_{\text {binned } \ell} \frac{(2 \ell+1) \Delta \ell f_{\text {sky }}}{2} \operatorname{Tr}\left[\mathbf{C}_{\ell}^{-1} \frac{\partial \mathbf{C}_{\ell}}{\partial \theta_{\alpha}} \mathbf{C}_{\ell}^{-1} \frac{\partial \mathbf{C}_{\ell}}{\partial \theta_{\beta}}\right]$,

where $\mathbf{C}_{\ell}$ is a $N_{\text {freq }} \times N_{\text {freq }}$ matrix ( $N_{\text {freq }}$ is the number of frequency bands) with its elements given by

$C_{\ell}{ }^{(i j)}=C_{\ell, v_{i} v_{j}}$,

where

$C_{\ell, v_{i} v_{j}}=C_{\ell, v_{i} v_{j}}^{\text {halo }}+C_{v_{i} v_{j}}^{\text {shot }}+C_{\ell, v_{i} v_{j}}^{\text {noise }} \delta_{i j}$.

The model parameters $\theta_{\alpha}$ correspond to the binned parameters $f_{i j}$ defined in Section 3.1. We use the same binning of $\ell$ as in P13 (see their table 4).

The fiducial value of each $f_{i j}$ is 0 , and we use a broad prior $\sigma_{i}=1$ for each parameter; that is, we add an identity matrix to the Fisher matrix. We then compute the eigenvalues $w_{\mu}$ of the Fisher matrix (sorted from large to small) and the corresponding eigenvectors $S_{\mu}$. The eigenvector associated with the largest eigenvalue is the first principal component, and it corresponds to the combination of parameters that is best constrained. The constraint on the $\mu$ th principal component is given by $\sigma_{\mu}=w_{\mu}^{-1 / 2}$. We define a principal component to be well constrained if it has a constraint $\sigma_{\mu}<0.1$.

To assess the constraining power of a given survey design, we define the figure of merit (FoM) using the determinant of the Fisher matrix,

$\log _{10}$ FoM $=\frac{\log _{10} \operatorname{det}\left(\mathbf{F}_{\text {survey }}\right)-\log _{10} \operatorname{det}\left(\mathbf{F}_{\text {Planck }}\right)}{30}$, 

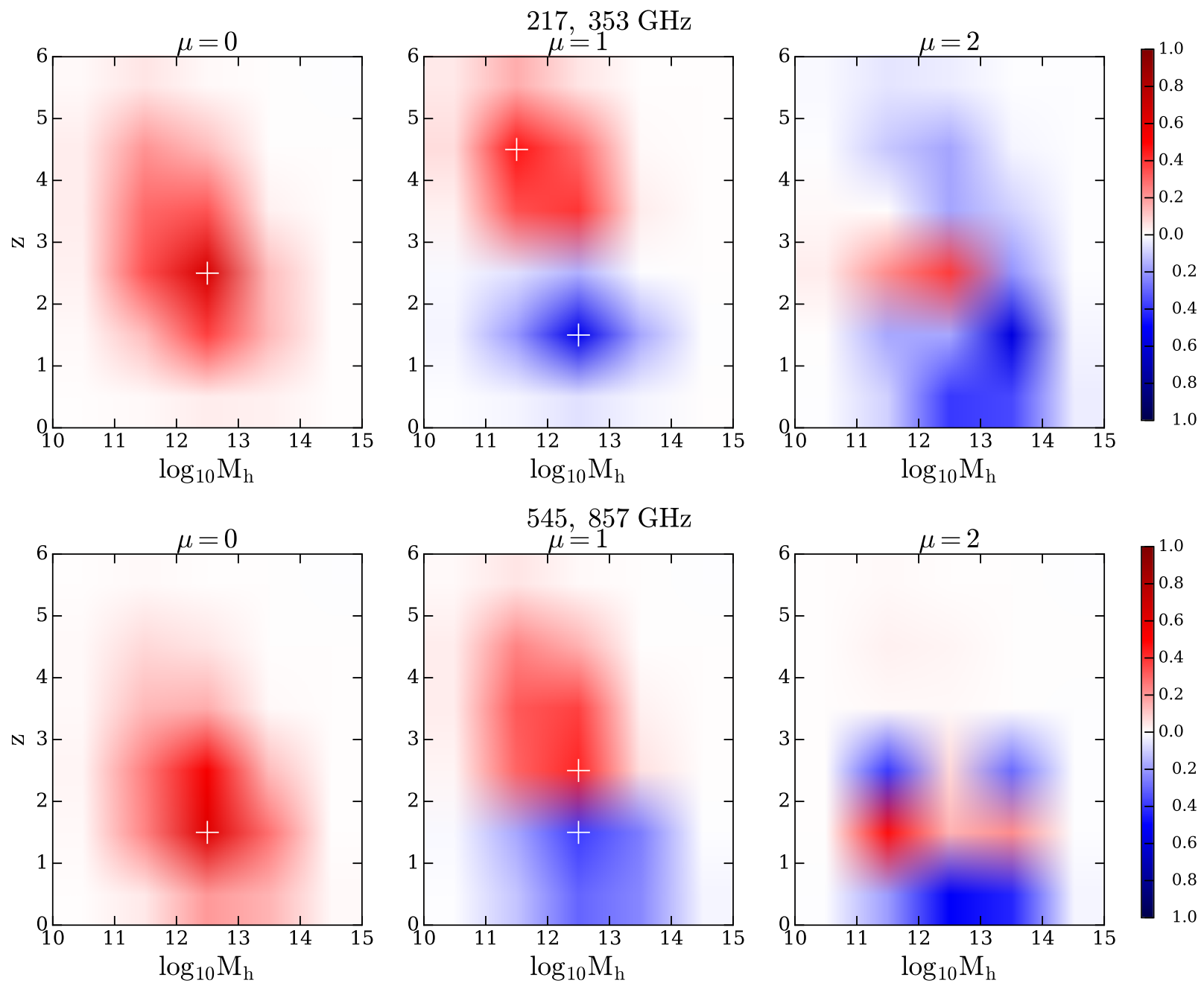

Figure 2. First three principal components of $\operatorname{SFR}(M, z)$ constrained by the CFIRB angular power spectra measured by Planck. Top row: the 217 and $353 \mathrm{GHz}$ bands. The best-constrained redshift lies between $z=2$ and 3. Bottom row: the 545 and $857 \mathrm{GHz}$ bands. The best-constrained redshift lies between $z=1$ and 2. In both cases, the best-constrained halo mass is between $10^{12}$ and $10^{13} \mathrm{M}_{\odot}$.

where the denominator 30 corresponds to the number of free parameters in our model. With this definition, $\log _{10} \mathrm{FoM}=1$ indicates that the constraints on $\operatorname{SFR}(M, z)$ parameters are on average improved by an order of magnitude.

\subsection{Principal components of SFR from Planck}

Fig. 2 presents the first three principal components of the $\operatorname{SFR}(M, z)$ parameters from the CFIRB power spectra measured by Planck. We arrange the 30 parameters on a two-dimensional grid, and the colour scheme shows the weight on each parameter (white represents 0 , and $\mathrm{red} / \mathrm{blue}$ represents positive/negative values). The top row corresponds to the principal components from the two low-frequency bands, 217 and $353 \mathrm{GHz}$, while the bottom row corresponds to the two high-frequency bands, 545 and $858 \mathrm{GHz}$. For the former, the peak of the first principal component is at $2<z<3$, while for the latter, the peak of the first component is at $1<z<2$. This trend confirms that CFIRB is dominated by galaxies between $1<z<3$ (e.g. Béthermin et al. 2013) and that the lower frequency bands probe higher redshifts. For both cases, the best-constrained halo mass is at $10^{12} \mathrm{M}_{\odot}<M<10^{13} \mathrm{M}_{\odot}$, which corresponds our assumption of $M_{\text {eff }}=10^{12.6} \mathrm{M}_{\odot}$ (see equation 4).
We note that the constraints from the high-frequency bands are stronger, and if we combine all four bands, the best-constrained redshift will be at $1<z<2$. For the same reason, if we further add the $3000 \mathrm{GHz}$ band from IRAS as P13 did, the first principal component will peak at $0<z<1$. Since the $3000 \mathrm{GHz}$ band mainly constrains SFR at $z<1$, we do not include this band in our work.

\section{OPTIMIZING THE SURVEY DESIGN}

We explore how the SFR constraints depend on the survey design. Our baseline survey assumption is as follows:

(i) Bands: 217, 353, 545, $857 \mathrm{GHz}(1382,849,550,350 \mu \mathrm{m})$

(ii) Survey area: $2240 \mathrm{deg}^{2}$

(iii) Detector noise: $\sigma_{\text {pix }}=0.015 \mathrm{MJy} \mathrm{sr}^{-1}$

Fig. 3 shows how the SFR constraints depend on these factors, and each row corresponds to varying one of these factors. In each panel, we present the SFR constraints ( $y$-axis) as a function of angular resolution ( $x$-axis, $\left.\ell_{\max }=\pi / \theta_{\mathrm{FWHM}}\right)$. The left-hand column corresponds to the number of well-constrained modes (with $\sigma_{\mu}<0.1$ ), while the right-hand column corresponds to the FoM 

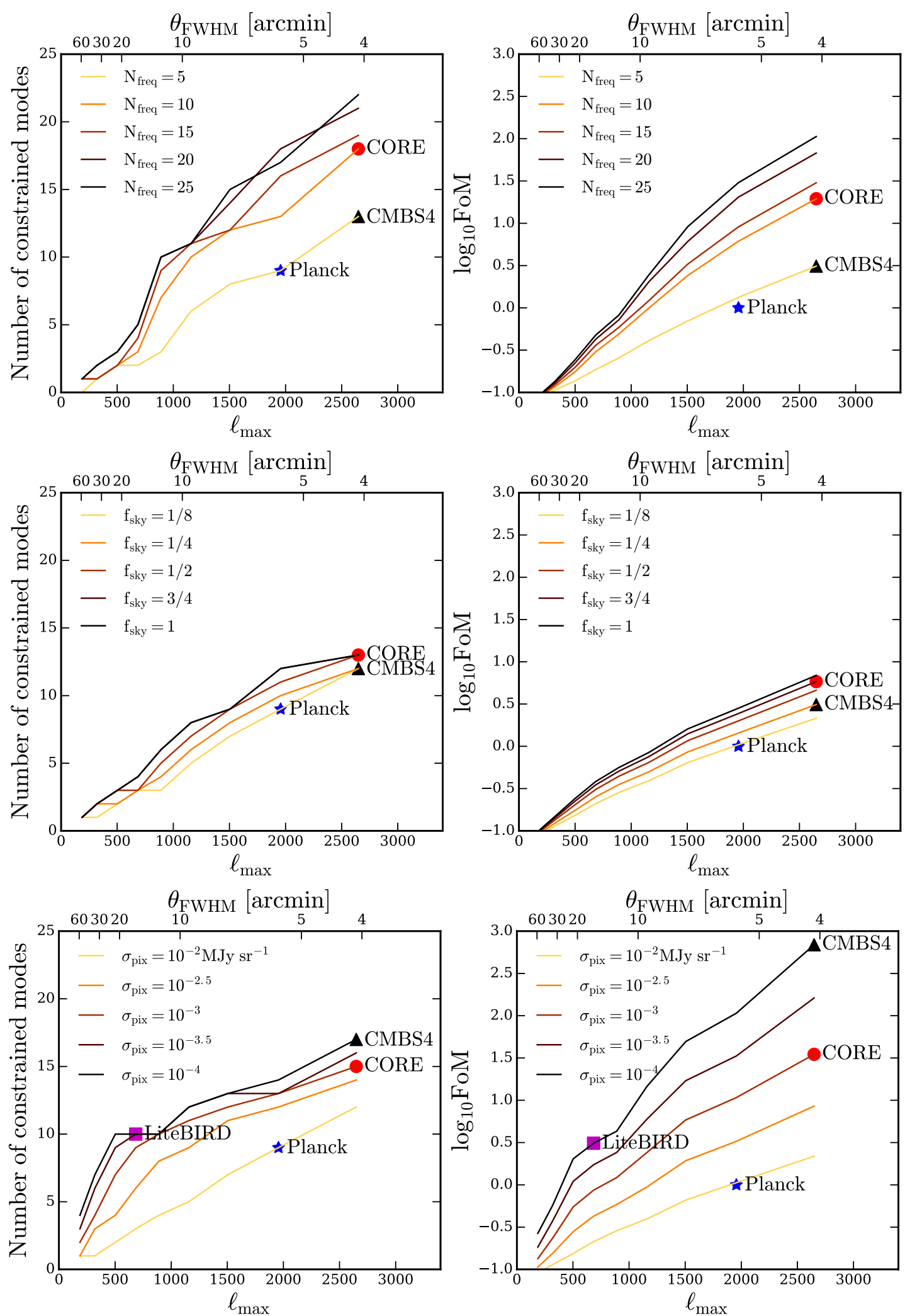

Figure 3. Impact of survey design on the $\operatorname{SFR}(M, z)$ constraints. Left: number of well-constrained modes versus angular resolution. Right: FoM (equation 26) versus angular resolution. The baseline survey assumption is presented in Section 4. Top: varying the number of frequency bands logarithmically spaced between 217 and $857 \mathrm{GHz}$. Middle: varying the sky coverage. Bottom: varying the instrumental sensitivity. As can be seen, improving the angular resolution and the instrumental sensitivity can significantly improve the SFR constraints. Increasing the number of frequency bands is only effective when the angular resolution is better than $\approx 20$ arcmin. In each panel, we mark the approximate loci for Planck, CORE, LiteBIRD and CMB-S4. We note that the CMB-S4 is likely to have a higher resolution than presented here and that LiteBIRD and CMB-S4 will only have 1 or 2 bands $(\lesssim 300 \mathrm{GHz})$ for CFIRB. 
Table 2. Designs of future experiments assumed in this work, adopted from The COrE Collaboration (2011, for CORE), Matsumura et al. (2014, for LiteBIRD) and Abazajian et al. (2016, for CMB-S4). The last column shows the improvement of SFR constraints compared with Planck; $\log _{10} \mathrm{FoM}=1$ corresponds to on average an order of magnitude improvement for SFR for all halo masses and redshifts.

\begin{tabular}{lccccc}
\hline Experiment & $\begin{array}{c}\theta_{\text {FWHM }} \\
(\operatorname{arcmin})\end{array}$ & $\begin{array}{c}\text { Frequency } \\
(\mathrm{GHz})\end{array}$ & $\begin{array}{c}\text { Sensitivity } \\
(\mu \text { K-arcmin })\end{array}$ & $\begin{array}{c}\text { Sensitivity improvement } \\
\text { w.r.t. Planck }\end{array}$ & $\begin{array}{c}\text { SFR constraints } \\
\log _{10} \text { FoM }\end{array}$ \\
\hline CORE & $1.3-4.7$ & $225-795(9$ bands $)$ & $27(315 \mathrm{GHz})$ & $\sim 10 \times$ & $1-1.5$ \\
LiteBIRD & 16 & 280 & 32 & $\sim 100 \times$ & 0.5 \\
CMB-S4 & $1-3$ & 250 & 1 & $\sim 100 \times$ & $0.5-3$ \\
\hline
\end{tabular}

defined in equation (26). We also mark the approximate loci for several experiments (see Table 2 and Section 6).

\subsection{Number of frequency bands}

The first row of Fig. 3 corresponds to varying the number of frequency bands $\left(N_{\text {freq }}\right)$ logarithmically spaced between 217 and $857 \mathrm{GHz}$. As can be seen, when the resolution is better than $\approx 20$ arcmin $\left(\ell_{\max } \gtrsim 500\right)$, increasing $N_{\text {freq }}$ from 5 to 10 leads to approximately an order of magnitude improvement in SFR constraints ( $\Delta \log _{10}$ FoM $\left.=1\right)$. The improvement with $N_{\text {freq }}$ is less significant beyond 10 bands, and the constraints saturate at approximately 20 bands. However, when the angular resolution is worse than $\approx 20$ arcmin, increasing the number of bands does not significantly improve the SFR constraints.

At a fixed $N_{\text {freq }}$, we can see that the SFR constraints sensitively depend on the angular resolution. For example, increasing the angular resolution from $\approx 20 \operatorname{arcmin}\left(\ell_{\max } \approx 500\right)$ to $\approx 4 \operatorname{arcmin}\left(\ell_{\max }\right.$ $\approx 2700$ ) can lead to 1.5 orders of magnitude improvement in SFR in the case of $N_{\text {freq }}=5$, and 2.5 orders of magnitude in the case of $N_{\text {freq }}=25$. Therefore, the angular resolution is more important than the number of bands in determining the constraining power of an experiment. The reason is as follows: the SED is a smooth function of frequency and redshift, and thus oversampling in frequency does not significantly increase the information content. Increasing the angular resolution, on the contrary, increases the number of multipole modes and can significantly improve the SFR constraints (see e.g. Wu \& Huterer 2013, for an analogy in the case of galaxy power spectrum)

The different designs of CORE (The COrE Collaboration 2011) and PIXIE (Kogut et al. 2011) provide an example of the trade-off between number of bands and angular resolution. While PIXIE is designed to densely sample the frequency space (400 bands from 30 to $6000 \mathrm{GHz}$ ) with a low angular resolution (2.6), CORE is designed to have fewer bands ( 15 bands from 45 to $795 \mathrm{GHz}$ ) with a higher angular resolution (1.3-4.7 arcmin). Based on our calculation, a survey design like PIXIE has limited constraining power for SFR, while a survey design like CORE can significantly improve the SFR constraints beyond Planck. In fact, PIXIE is optimized for precise measurement of the absolute intensity and linear polarization of CMB rather than for measurement of SFR, and these two measurements require very different survey strategies.

\subsection{Sky coverage}

The second row of Fig. 3 corresponds to varying the fraction of the sky coverage, $f_{\text {sky }}$. We show SFR constraints for $f_{\text {sky }}=1 / 8$, $1 / 4,1 / 2,3 / 4$ and 1 , as a function of angular resolution. As can be seen, the improvement due to increased sky coverage is relatively modest compared with increasing angular resolution or $N_{\text {freq. }}$. For example, increasing the sky coverage from $1 / 8$ to full sky will lead to approximately half an order of magnitude improvement in SFR constraints.

We note that the Planck 2013 CFIRB results are based on a survey area of $2240 \mathrm{deg}^{2}\left(f_{\text {sky }} \approx 0.05\right)$. This sky coverage is limited by the radio measurements for the neutral atomic hydrogen $(\mathrm{H} \mathrm{I}) \mathrm{col}-$ umn density, which are required to remove the foreground Galactic dust. Increasing the sky coverage will require new radio surveys and thus substantial extra observational resources (e.g. HI4PI Collaboration 2016); in this sense, increasing the sky coverage may not be the most efficient way for improving the SFR constraints from CFIRB. Nevertheless, increasing the sky coverage and observing different regions of the sky is still invaluable because it provides essential consistency checks and controls systematic errors.

\subsection{Instrumental sensitivity}

The third row of Fig. 3 corresponds to different instrumental sensitivities. We characterize the sensitivity using the detector noise $\left(\sigma_{\text {pix }}\right)$ and the angular resolution $\left(\theta_{\text {FWHM }}\right)$ introduced in Section 2.4. For $\sigma_{\text {pix }}$, we use the intensity unit $\mathrm{MJy} \mathrm{sr}^{-1}$; in Table 1 , we present the conversion between $\mathrm{MJy} \mathrm{sr}^{-1}$ and $\mu \mathrm{K}_{\mathrm{CMB}}$, as well as the sensitivity in the commonly used unit $\mu \mathrm{K}_{\mathrm{CMB}}$-arcmin for the Planck bands. In the last two columns of Table 1, we list two hypothetical experiments with given $\sigma_{\text {pix }}$ and $\theta_{\text {FWHM }}$, and we calculate the corresponding sensitivity in the unit of $\mu \mathrm{K}_{\mathrm{CMB}}$-arcmin assuming the bandpass filters of Planck. For other combinations of $\sigma_{\text {pix }}$ and $\theta_{\mathrm{FWHM}}$, the sensitivity can be estimated by rescaling the numbers in the last two columns.

We assume that all frequency bands have the same sensitivity, and we show the cases of $\sigma_{\mathrm{pix}}=10^{-2}, 10^{-2.5}, 10^{-3}, 10^{-3.5}$ and $10^{-4} \mathrm{MJy} \mathrm{sr}^{-1}$. For comparison, Planck has $\sigma_{\text {pix }}=0.0155 \mathrm{MJy} \mathrm{sr}^{-1}$ at $857 \mathrm{GHz}$. As can be seen, reducing the pixel noise can significantly improve the SFR constraints; for example, reducing the pixel noise by two orders of magnitude can improve the SFR constraints by $1-2.5$ orders of magnitude, and the improvement is greater with a higher angular resolution.

We can only roughly estimate the pixel noise levels for future experiments. In Table 2, we list the targeted sensitivities for several experiments, taken from The COrE Collaboration (2011, for CORE), Matsumura et al. (2014, for LiteBIRD) and Abazajian et al. (2016, for CMB-S4). From these values, we estimate that CORE will have a sensitivity $\sim 10$ time better than Planck and that Lite$B I R D$ andCMB-S4 will have sensitivities $\sim 100$ times better than Planck. In Fig. 3, we mark the approximate loci of these experiments accordingly. The CMB-S4 is more likely to have even higher angular resolution and sensitivity than presented in our figure.

We note that CMB-S4 and LiteBIRD will include only the lowfrequency bands for CFIRB $(\lesssim 300 \mathrm{GHz})$. Although these bands have limited information for SFR at $z \lesssim 2$, they will provide 

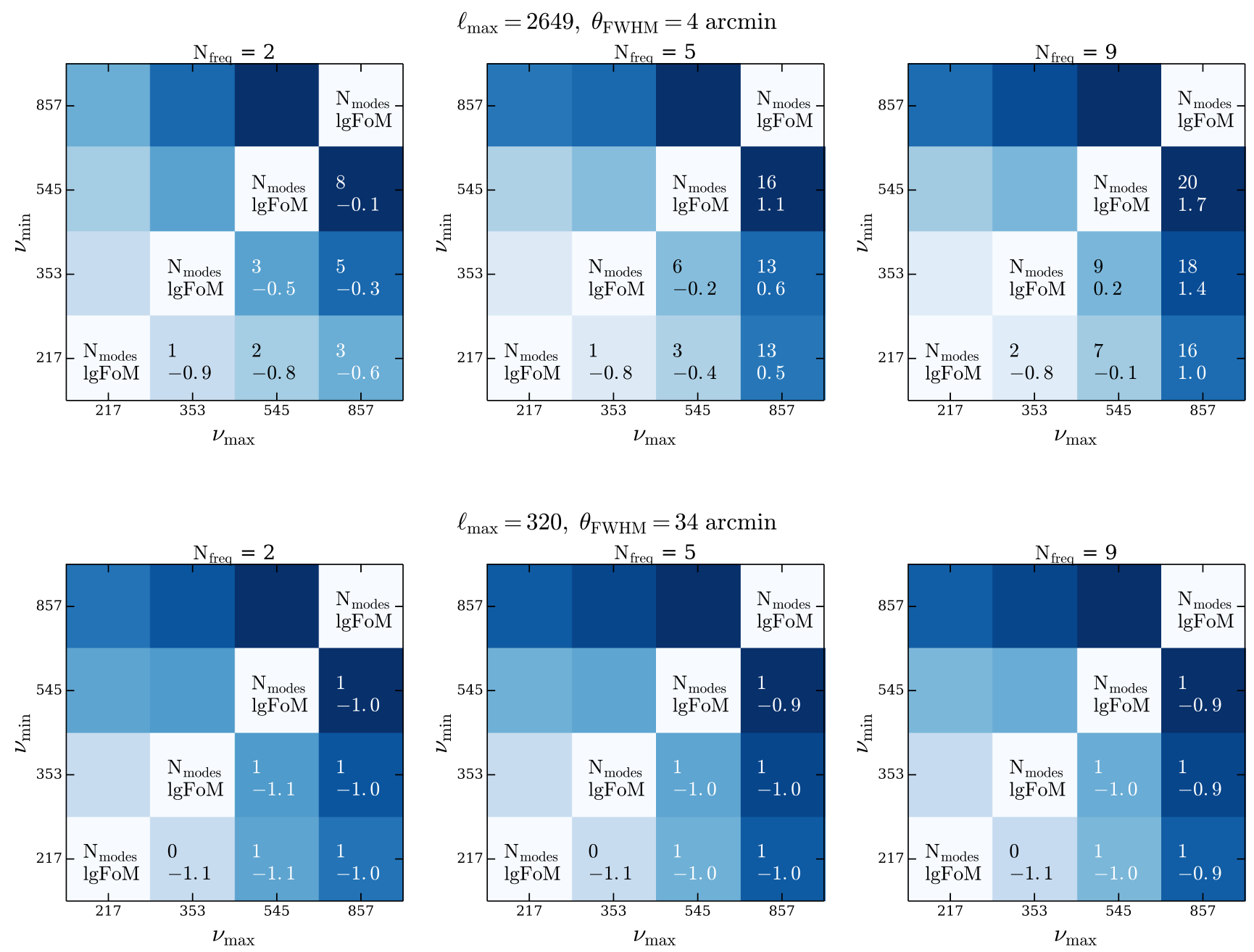

Figure 4. Comparison of various ranges and numbers of frequency bands. The top/bottom row corresponds to high/low angular resolution. The lefthand/central/right-hand column corresponds to $2 / 5 / 9$ bands logarithmically spaced between $v_{\min }$ ( $y$-axis) and $v_{\max }$ ( $x$-axis). In each cell, the shading corresponds to the $\log _{10}$ FoM, and we show the number of well-constrained modes and the $\log _{10}$ FoM. The high-frequency bands can constrain more modes, but these modes are limited to low redshift. When the angular resolution is high, increasing the number of bands is effective in improving the SFR constraints, and the best improvement is when $v_{\min }$ and $v_{\max }$ span a wide range. However, when the angular resolution is low, increasing the number of bands cannot significantly improve SFR constraints.

constraints on high-redshift galaxies $(z \gtrsim 3)$. Such constraints will be invaluable, because at $z \gtrsim 3$, CFIRB may never be completely resolved into individual galaxies. However, at these low-frequency bands, the power spectrum is dominated by $\mathrm{CMB}$, and separating $\mathrm{CMB}$ and CFIRB can be a major challenge (see P13).

\section{OPTIMAL RANGE OF FREQUENCY BANDS}

In this section, we explore the SFR constraints from various ranges of frequency bands. We use $\left(v_{\min }, v_{\max }\right)$ pairs selected from the Planck bands: $(217,353,545,857) \mathrm{GHz}$, and we assume $N_{\text {freq }}$ logarithmically spaced bands between $v_{\min }$ and $v_{\max }$. We adopt the same baseline survey design as in Section 4.

Fig. 4 shows the comparison between different $N_{\text {freq }}$ and ( $v_{\min }$, $\left.v_{\max }\right)$. The top row corresponds to a high-resolution survey with $\theta_{\text {FWHM }}=4 \operatorname{arcmin}\left(\ell_{\max }=2649\right)$, while the bottom row corresponds to a low-resolution survey with $\theta_{\mathrm{FWHM}}=34 \operatorname{arcmin}\left(\ell_{\max }=320\right)$. The left-hand, central and right-hand panels correspond to $N_{\text {freq }}=2$, 5 and 9 . For each panel, the $y$-axis corresponds to $v_{\min }$, and the $x$-axis corresponds to $v_{\max }$. In each cell, the two numbers correspond to the number of well-constrained modes and the $\log _{10}$ FoM from the given frequency bands, and the shading is based on $\log _{10}$ FoM.

From the top row, we can see that for a given number of bands, the high-frequency bands always constrain the largest number of modes. However, as shown in Fig. 2, the high-frequency bands only constrain SFR at low redshift. In order to constrain SFR at high redshift, we need low-frequency bands, but the improvement is rather slow when we increase the number of bands between 217 and $353 \mathrm{GHz}$. On the other hand, if we compare the improvements associated with increased $N_{\text {freq }}$, we can see that the cells correspond to $(217,857)$ and $(353,857)$ show the most significant improvement; that is, spanning a wide range of frequencies can be beneficial.

From the bottom row, we can see that the constraining power of an experiment is significantly reduced when the resolution is low. The FoM values are low with this resolution, and increasing the number of bands barely improves the constraining power of the experiment. Comparing the top and bottom panels, we can once again see that it is more important to improve the angular resolution than to increase the number of bands for constraining SFR. 


\section{IMPLICATIONS FOR FUTURE CFIRB EXPERIMENTS}

In this section, we put our results in the context of several future experiments that are currently being planned. We note that most of these experiments are optimized for measuring $\mathrm{CMB}$ polarization, and they include FIR/submm bands for measuring dust emissions in order to control systematics. These bands will contain rich information for extragalactic astrophysics, and the CFIRB measured from these experiments will significantly improve the constraints on the cosmic star formation history. Below we compare the survey designs of several experiments (also see Table 2), and we note that the design we quote below are only approximate and are still evolving.

The CORE mission is the next-generation space-borne CMB experiment, which is proposed to European Space Agency and designed to be the successor of Planck. The major improvement beyond Planck is the high sensitivity and a diffraction-limited resolution, as well as twice as many frequency bands as Planck. One of the experimental designs, as presented in The COrE Collaboration (2011), includes 15 frequency bands between $45 \mathrm{GHz}(6.7 \mathrm{~mm})$ and $795 \mathrm{GHz}(377 \mu \mathrm{m})$, and the angular resolution ranges from 23 arcmin at $45 \mathrm{GHz}$ to $1.3 \operatorname{arcmin}$ at $795 \mathrm{GHz}$. In addition, the sensitivity of CORE is expected to be 10-30 times better than that of Planck. As we have shown in Section 4, such a survey design is very close to optimal for constraining SFR.

The CORE team has recently published several designs, including a mirror ranging from 1 to $1.5 \mathrm{~m}$ and $v_{\max }$ from 600 to $800 \mathrm{GHz}$ (De Zotti et al. 2015, 2016; Di Valentino et al. 2016). Based on our results, if one has to choose between the number of bands and the angular resolution, it is the latter that will provide stronger SFR constraints. We note that the extragalactic sources that will be resolved by CORE have been studied in De Zotti et al. (2015, 2016). Our work is highly complementary to those studies in the sense that we focus the SFR constraints of faint, unresolved galaxies.

The LiteBIRD mission is another next-generation space-borne $\mathrm{CMB}$ experiment, which is designed to cover the frequency range from 50 to $320 \mathrm{GHz}$, with a sensitivity of $2 \mu \mathrm{K}$-arcmin and an angular resolution of 16 arcmin (Matsumura et al. 2014). The sensitivity is superior to CORE, while the angular resolution is lower. As we have shown in Section 4, such a high sensitivity is beneficial for improving the SFR constraints from CFIRB. The highest frequency is relatively low for CFIRB, but it can be beneficial for constraining SFR at high redshift.

The next-generation ground-based CMB-S4 experiment will have superior angular resolution ( $\lesssim 1$ arcmin) and a sensitivity two orders of magnitude better than Planck (Abazajian et al. 2016). Operating from the ground, CMB-S4 can only observe through the atmospheric windows, and the highest frequency is around $250 \mathrm{GHz}$. Therefore, CMB-S4 will provide CFIRB measurements at low frequencies and constrain the SFR at $z \gtrsim 3$. In addition, CMB-S4 will measure $\mathrm{CMB}$ lensing to unprecedented precision, enabling detailed studies of the cross-correlation between CFIRB and CMB lensing potential. This correlation is essential for probing the connection between SFR and halo mass and providing consistency checks for the CFIRB power spectra. Nevertheless, at $\lesssim 250 \mathrm{GHz}$, separating CFIRB from CMB can be a major challenge, and careful component separation will be required to extract CFIRB to high precision.

The PIXIE (Kogut et al. 2011) is a space-borne CMB mission designed for measuring the large-scale polarization. It will significantly improve the precision of the measurement of the absolutely intensity of the cosmic background radiation compare with $C O B E$. The survey design includes 400 effective channels from $30 \mathrm{GHz}$ $(10 \mathrm{~mm})$ to $6 \mathrm{THz}(50 \mu \mathrm{m})$, with an angular resolution of 2.6. Despite its unique role in measuring the absolute intensity of CFIRB, it has limited angular resolution to measure the anisotropies of CFIRB; therefore, as we have demonstrated, it is not optimal for constraining SFR.

In addition, FIR/submm survey telescopes with wide field of view have also been planned, including the Cerro Chajnantor Atacama Telescope (CCAT, Woody et al. 2012) and the Far-infrared Surveyor (Origins Space Telescope; Meixner et al. 2016). These telescopes usually operate at frequencies higher than CMB telescopes ( $\gtrsim 500 \mathrm{GHz}$ ) and will also measure CFIRB in much smaller area of the sky. More importantly, these telescopes will have the power to resolve a significant fraction of the sources contributing to CFIRB at low redshift, and thus they are complementary to the CFIRB experiments discussed in this work.

\section{SUMMARY}

We explore the optimal survey strategies for future CFIRB experiments, and our goal is to maximize the SFR constraints extracted from the CFIRB angular power spectra. We introduce a modelindependent, piecewise parametrization for $\operatorname{SFR}(M, z)$, and we calculate the Fisher matrix to estimate how well future experiments can constrain SFR. Our findings are summarized as follows:

(i) From the first principal component of the Fisher matrix, we find that for the CFIRB power spectra observed by Planck, the 217 and $353 \mathrm{GHz}$ bands (1382 and $849 \mu \mathrm{m})$, provide the strongest SFR constraints at $2<z<3$, while the 545 and $857 \mathrm{GHz}$ bands $(550$ and $350 \mu \mathrm{m}$ ) provide the strongest SFR constraints at $1<z<2$. In all redshifts, the strongest SFR constraints are associated with halo mass between $M=10^{12}$ and $10^{13} \mathrm{M}_{\odot}$.

(ii) We find that the angular resolution and the instrumental sensitivity are the most important factors for improving SFR constraints. For example, improving the angular resolution from 20 to 4 arcmin can lead to 1.5-2.5 orders of magnitude improvement in SFR constraints. In addition, improving the sensitivity by 10 or 100 times with respect to Planck can lead to one to two orders of magnitude improvement in SFR constraints, and the improvement is greater when the angular resolution is higher. We also find that increasing the survey area only leads to modest improvement in SFR constraints.

(iii) Increasing the number of frequency bands is not always effective in improving SFR constraints. With an angular resolution similar to Planck ( $\approx 5$ arcmin), doubling the number of frequency bands of Planck can improve SFR constraints by an order of magnitude. Further increasing the number of bands has less impact on SFR constraints, and the constraints saturate at approximately 20 bands. However, if the angular resolution is lower than 20 arcmin, increasing the number of bands can hardly improve the SFR constraints. Therefore, in terms of maximizing the SFR constraints, a survey design with high angular resolution and relatively fewer bands (e.g. $C O R E$ ) is favoured over a design with low angular resolution and a large number of bands (e.g. PIXIE).

(iv) We explore the constraining power of various ranges and numbers of frequency bands. With an angular resolution similar to Planck, we find that the SFR constraints improve relatively slowly when we increase the number of low-frequency bands; that is, it is relatively difficult to improve SFR constraints at high redshift. If we consider SFR constraints for all redshifts, spanning a wide range of 
frequencies will lead to the most significant improvement. On the other hand, when the resolution is low ( $\lesssim 20$ arcmin), increasing the number of bands in any frequency range has very limited impact on SFR constraints.

(v) When comparing our results with the designs of several future CMB missions, we find that CORE has nearly the optimal angular resolution and number of bands to significantly improve SFR constraints. In addition, LiteBIRD will have superior sensitivity, and CMB-S4 will have both superior sensitivity and high angular resolution. Since LiteBIRD and CMB-S4 will only cover low frequencies $(\lesssim 300 \mathrm{GHz})$, they will mainly constrain the SFR at high redshift. The proposed Far-Infrared Surveyor will have superior angular resolution and sensitivity, but it will focus on high frequencies and smaller survey area; thus, it will efficiently resolve sources contributing to CFIRB and constrain the SFR at low redshift. The design of PIXIE is optimized for large-scale polarization measurements and is not optimal for constraining SFR.

In this work, we only consider the constraints of SFR from CFIRB angular power spectra. We expect that other FIR/submm observations will provide extra SFR constraints and important consistency checks. These observations include the IR luminosity functions (e.g. Gruppioni et al. 2013), submm number counts (e.g. Béthermin et al. 2013; Valiante et al. 2016), the cross-correlation between CFIRB and CMB lensing potential (e.g. Planck Collaboration XVIII 2014) and the cross-correlation between CFIRB and other extragalactic background light (e.g. Cooray 2016). Among these observations, CFIRB is still a powerful and unique probe because it provides the rare opportunity to constrain the star formation activities under the current resolution limit. A robust measurement of CFIRB will require not only superior angular resolution and sensitivity of instruments but also careful control of systematic errors and comprehensive cross-correlation studies.

\section{ACKNOWLEDGEMENTS}

HW acknowledges the support by the US National Science Foundation (NSF) grant AST1313037. The calculations in this work were performed on the Caltech computer cluster Zwicky, which is supported by NSF MRI-R2 award number PHY-096029. Part of the research described in this paper was carried out at the Jet Propulsion Laboratory, California Institute of Technology, under a contract with the National Aeronautics and Space Administration.

\section{REFERENCES}

Abazajian K. N. et al., 2016, preprint (arXiv:1610.02743)

Addison G. E., Dunkley J., Bond J. R., 2013, MNRAS, 436, 1896

Amblard A. et al., 2011, Nature, 470, 510

Berta S. et al., 2011, A\&A, 532, A49

Béthermin M., Wang L., Doré O., Lagache G., Sargent M., Daddi E., Cousin M., Aussel H., 2013, A\&A, 557, A66

Bhattacharya S., Habib S., Heitmann K., Vikhlinin A., 2013, ApJ, 766, 32 Bock J. et al., 2009, preprint (arXiv:0906.1188)

Bond J. R., Carr B. J., Hogan C. J., 1986, ApJ, 306, 428

Casey C. M., Narayanan D., Cooray A., 2014, Phys. Rep., 541, 45

Chary R., Elbaz D., 2001, ApJ, 556, 562

Cooray A., 2016, R. Soc. Open Sci., 3, 150555

Cowley W. I., Lacey C. G., Baugh C. M., Cole S., 2016, MNRAS, 461, 1621

De Bernardis F., Cooray A., 2012, ApJ, 760, 14

De Zotti G. et al., 2015, J. Cosmol. Astropart. Phys., 6, 018

De Zotti G. et al., 2016, preprint (arXiv:1609.07263)

Di Valentino E. et al., 2016, preprint (arXiv:1612.00021)

Dole H. et al., 2006, A\&A, 451, 417
Elbaz D., Cesarsky C. J., Chanial P., Aussel H., Franceschini A., Fadda D., Chary R. R., 2002, A\&A, 384, 848

Fixsen D. J., Dwek E., Mather J. C., Bennett C. L., Shafer R. A., 1998, ApJ, 508,123

Gruppioni C. et al., 2013, MNRAS, 432, 23

Hajian A. et al., 2012, ApJ, 744, 40

Hall N. R. et al., 2010, ApJ, 718, 632

Hauser M. G., Dwek E., 2001, ARA\&A, 39, 249

Hauser M. G. et al., 1998, ApJ, 508, 25

HI4PI Collaboration, 2016, A\&A, 594, A116

Huterer D., Starkman G., 2003, Phys. Rev. Lett., 90, 031301

Kennicutt R. C., Jr, 1998, ApJ, 498, 541

Knox L., 1995, Phys. Rev. D, 52, 4307

Knox L., Cooray A., Eisenstein D., Haiman Z., 2001, ApJ, 550, 7

Kogut A. et al., 2011, J. Cosmol. Astropart. Phys., 7, 25

Lagache G., Puget J.-L., Dole H., 2005, ARA\&A, 43, 727

Lagache G., Bavouzet N., Fernandez-Conde N., Ponthieu N., Rodet T., Dole H., Miville-Deschênes M.-A., Puget J.-L., 2007, ApJ, 665, L89

Lewis A., Challinor A., Lasenby A., 2000, ApJ, 538, 473

Lutz D., 2014, ARA\&A, 52, 373

Madau P., Dickinson M., 2014, ARA\&A, 52, 415

Matsumura T. et al., 2014, J. Low Temp. Phys., 176, 733

Matsuura S. et al., 2011, ApJ, 737, 2

Meixner M. et al., 2016, in MacEwen H. A., Fazio G. G., Lystrup M., eds, Proc. SPIE Conf. Ser. Vol. 9904, Space Telescopes and Instrumentation 2016: Optical, Infrared, and Millimeter Wave. SPIE, Bellingham, p. $99040 \mathrm{~K}$

Mortonson M. J., Hu W., 2008, ApJ, 672, 737

Navarro J. F., Frenk C. S., White S. D. M., 1997, ApJ, 490, 493

Planck Collaboration XVIII, 2011, A\&A, 536, A18

Planck Collaboration I, 2014, A\&A, 571, A1

Planck Collaboration XVIII, 2014, A\&A, 571, A18

Planck Collaboration XXX, 2014, A\&A, 571, A30 (P13)

Puget J.-L., Abergel A., Bernard J.-P., Boulanger F., Burton W. B., Desert F.-X., Hartmann D., 1996, A\&A, 308, L5

Shang C., Haiman Z., Knox L., Oh S. P., 2012, MNRAS, 421, 2832 (S12)

Thacker C. et al., 2013, ApJ, 768, 58

The COrE Collaboration, 2011, preprint (arXiv:1102.2181)

Tinker J. L., Wetzel A. R., 2010, ApJ, 719, 88

Tinker J. L., Robertson B. E., Kravtsov A. V., Klypin A., Warren M. S., Yepes G., Gottlöber S., 2010, ApJ, 724, 878

Valiante E. et al., 2016, MNRAS, 462, 3146

Viero M. P. et al., 2009, ApJ, 707, 1766

Viero M. P. et al., 2013, ApJ, 772, 77

Woody D. et al., 2012, in Stepp L. M., Gilmozzi R., Hall H. J., eds, Proc. SPIE Conf. Ser. Vol. 8444, Ground-based and Airborne Telescopes IV. SPIE, Bellingham, p. 84442M

Wu H.-Y., Doré O., 2017, MNRAS, 466, 4651

Wu H.-Y., Huterer D., 2013, MNRAS, 434, 2556

Wu H.-Y., Doré O., Teyssier R., 2016, preprint (arXiv:1607.02546)

Xia J.-Q., Negrello M., Lapi A., De Zotti G., Danese L., Viel M., 2012, MNRAS, 422, 1324

\section{APPENDIX A: DERIVATION OF SHOT NOISE}

The spectral flux is given by

$S_{v}=\frac{L_{(1+z) v}}{4 \pi \chi^{2}(1+z)}=S(1+z) \Theta_{(1+z) v}$

where $S$ is the bolometric flux given by

$S=\frac{L}{4 \pi \chi^{2}(1+z)^{2}}$. 
The shot noise of the auto power spectrum is given by the integration

$$
\begin{aligned}
C_{v v}^{\text {shot }} & =\int \mathrm{d} V \int_{S_{v}^{\min }}^{S_{v}^{\max }} \mathrm{d} S_{v} \frac{\mathrm{d} n}{\mathrm{~d} S_{v}} S_{v}^{2} \\
& =\int \mathrm{d} V \int_{S^{\min }}^{S^{\max }} \mathrm{d} S \frac{\mathrm{d} n}{\mathrm{~d} S} S^{2}(1+z)^{2} \Theta_{(1+z) v}^{2},
\end{aligned}
$$

where

$\mathrm{d} V=\frac{D_{H}}{E(z)} \chi^{2} \mathrm{~d} z \mathrm{~d} \Omega$.

The upper limit of the integration, $S_{v}^{\max }$, corresponds to the flux cut of the observation, and we adopt the values in table 1 of P13. In practice, our model includes negligible number of galaxies above the flux cut because we do not include starburst or lensed galaxies.

The shot noise of the cross power spectrum is given by

$C_{\nu v^{\prime}}^{\text {shot }}=\int \mathrm{d} V \int_{S^{\min }}^{S^{\max }} \mathrm{d} S \frac{\mathrm{d} n}{\mathrm{~d} S} S^{2}(1+z)^{2} \Theta_{(1+z) \nu} \Theta_{(1+z) v^{\prime}}$.

To calculate $\mathrm{d} n / \mathrm{d} S(z)$, we integrate over the probability distribution function of $S$ at a given $M$,

$\frac{\mathrm{d} n}{\mathrm{~d} \ln S}=\int \mathrm{d} \ln M \frac{\mathrm{d} n}{\mathrm{~d} \ln M} P(\ln S \mid \ln M)$,

where we assume $P(\ln S \mid \ln M)$ follows a normal distribution

$$
P(\ln S \mid \ln M) \sim \operatorname{Normal}\left(\langle\ln S(M)\rangle ; \sigma^{2}\right),
$$

with the mean given by

$$
\langle\ln S(M)\rangle=\ln \langle S(M)\rangle-\frac{\sigma^{2}}{2},
$$

where

$$
\langle S(M, z)\rangle=\frac{\left\langle L_{\mathrm{IR}}(M, z)\right\rangle}{4 \pi \chi^{2}(1+z)} .
$$

We find that a scatter of $\sigma=0.9(0.28 \mathrm{dex})$ is required to reproduce the shot noise in P13. We note that this scatter does not affect any of the equations presented in Section 2.2, because those questions only involve $\left\langle L_{\mathrm{IR}}\right\rangle$. We also find that satellite galaxies have a negligible contribution to the shot noise.

This paper has been typeset from a $\mathrm{T}_{\mathrm{E}} \mathrm{X} / \mathrm{LT}_{\mathrm{E}} \mathrm{X}$ file prepared by the author. 